\title{
BEHAVIOR OF POLYNOMIALS OF BEST UNIFORM APPROXIMATION
}

\author{
E. B. SAFF AND V. TOTIK
}

\begin{abstract}
We investigate the asymptotic behavior of the polynomials $\left\{P_{n}(f)\right\}_{0}^{\infty}$ of best uniform approximation to a function $f$ that is continuous on a compact set $K$ of the complex plane $\mathbf{C}$ and analytic in the interior of $K$, where $K$ has connected complement. For example, we show that for "most" functions $f$, the error $f-P_{n}(f)$ does not decrease faster at interior points of $K$ than on $K$ itself. We also describe the possible limit functions for the normalized error $\left(f-P_{n}(f)\right) / E_{n}$, where $E_{n}:=\left\|f-P_{n}(f)\right\|_{K}$, and the possible limit distributions of the extreme points for the error. In contrast to these results, we show that "near best" polynomial approximants to $f$ on $K$ exist that converge more rapidly at the interior points of $K$.
\end{abstract}

\section{INTRODUCTION}

Let $K$ be a compact subset of $\mathbf{C}$ consisting of infinitely many points, the complement of which is connected. The set of continuous functions on $K$ that are analytic in the interior $K^{0}$ of $K$ will be denoted by $A(K)$. When $K=\bar{U}:=\{z:|z| \leq 1\}$ is the closed unit disk, then $A(K)$ is the classical disk algebra $\mathscr{A}$. If $\Pi_{n}$ is the set of polynomials in $z$ of degree at most $n$, then

$$
E_{n}(f):=\inf _{P_{n} \in \Pi_{n}}\left\|f-P_{n}\right\|_{K}
$$

is the error in the best uniform approximation of $f$ out of $\Pi_{n}$; the unique best approximant (i.e., the unique $P_{n} \in \Pi_{n}$ minimizing $\left\|f-P_{n}\right\|$ ) is denoted by $P_{n}(f, z)$. Here and in what follows $\|\cdot\|=\|\cdot\|_{K}$ always denotes the supremum norm.

By a basic result of Mergelyan (cf. [12]), $E_{n}(f) \rightarrow 0$ as $n \rightarrow \infty$ for every $f \in A(K)$. Furthermore, under mild assumptions on $K,\left\{E_{n}(f)\right\}_{n=0}^{\infty}$ tends to zero geometrically if and only if $f$ is analytic on $K$ (cf. [20, $\S 4.7]$ ). We will primarily be interested in the behavior of the normalized error

$$
Q_{n}(f, z):=\left(f(z)-P_{n}(f, z)\right) / E_{n}(f),
$$

Received by the editors April 28, 1988.

1980 Mathematics Subject Classification (1985 Revision). Primary 41A50.

The research of E. B. Saff was supported, in part, by the National Science Foundation under grant DMS-862-0098.

The research of V. Totik was partially supported by the Hungarian National Science Foundation for Research, Grant No. 1157. This work was done while he visited the University of South Florida, Tampa. 
and let us agree that if $E_{n}(f)=0$ for a given $n$, i.e., when $f$ is a polynomial of degree at most $n$, then we set $Q_{n}(f, z) \equiv 0$. Clearly, $\left|Q_{n}(f, z)\right| \leq 1$ for $z \in K$, and $\left\{Q_{n}(f)\right\}_{n=0}^{\infty}$ forms a normal family on $K^{0}$.

It was shown in [9] that every point on the boundary $\partial K$, except possibly for points in a set of zero capacity, is a limit point of the sets

$$
H_{n}(f):=\left\{z \in K:\left|Q_{n}(f, z)\right|=1\right\}
$$

consisting of the extreme points of the $n$th best polynomial approximation. Thus, on the boundary of $K, Q_{n}(f, z)$ cannot be expected to be small (for large $n$ ), but it may happen that on compact sets in the interior $K^{0}$ of $K$ the approximation given by $P_{n}(f, z)$ is better than on $K$, which would mean that $\left\{Q_{n}(f, z)\right\}_{n=0}^{\infty}$ converges to zero in the interior of $K$. However, it turns out that this is very exceptional, and one aim of this paper is to describe the possible limiting behavior of the error functions $Q_{n}(f, z)$ and the extremal sets $H_{n}(f)$.

There are several motivations for this paper beyond the interest in exploring the behavior of the best polynomial approximants on general compact sets. The first is to construct "better than best" approximants in the sense that, where permitted (say inside the domains of analyticity), the approximations do better than what is possible on the whole of $K$ (see Theorems 2 and 4 below). We proved the first result in this direction in [15], where we verified that if $f \in$ $C^{k}[-1,1]$ is piecewise analytic, then there are polynomials $p_{n} \in \Pi_{n}, n=$ $1,2, \ldots$, such that

$$
\left\|f-p_{n}\right\|_{[-1,1]} \leq C n^{-k-1},
$$

and, at every point of $[-1,1]$ where $f$ is analytic,

$$
\varlimsup_{n \rightarrow \infty}\left|f(x)-p_{n}(x)\right|^{1 / n}<1 \text {. }
$$

Furthermore, (1.3) holds uniformly on compact subsets of $[-1,1]$ where $f$ is analytic (in a neighborhood of a singularity of $f$ inequality (1.3) is impossible). In [15] we also determined the best upper bound for the left-hand side of (1.3).

The second motivation emanates from a result of [4]. In a special case, this result says that if $w$ is any continuous weight function on the closed unit disk $\bar{U}$ that is positive on $U$ (otherwise $w$ may vanish identically on the boundary $\partial U)$, and if $P_{n, w}(f) \in \Pi_{n}$ satisfies

$$
\left\|w\left(f-P_{n, w}(f)\right)\right\|_{\overline{U^{u}}}=\inf _{P_{n} \in \Pi_{n}}\left\|w\left(f-P_{n}\right)\right\|_{\bar{U}}=: E_{n}(f)_{w},
$$

then for $f \in A(\bar{U})$ every point on $\partial U$ is a limit point of the sets

$$
H_{n}(f)_{w}:=\left\{z \in \bar{U}: w(z)\left|f(z)-P_{n, w}(f, z)\right|=E_{n}(f)_{u}\right\} .
$$

If, for example, $H_{n}(f)_{w}$, consists of $(n+2)$ points (it must contain at least this many) then (for some subsequence of integers $n$ ) all the extrema of $\left|w(z)\left(f(z)-P_{n, w}(f, z)\right)\right|$ occur near $\partial U$. This is rather surprising in view of the fact that $w$ is merely assumed to be continuous, not analytic. One conceivable explanation for this phenomenon might be that best approximants give 
much better approximation inside $K^{0}$ than on $K$. However, we will see below that this is not the case, even for $w \equiv 1$ (cf. Theorems 1 and 5).

Another motivation for our investigation is the important result of Kadec [7] concerning the distribution of maximal alternation sets in real polynomial approximation on an interval. Considerably generalizing this result, it is shown in [4] that if $H_{n}(f)$ (cf. (1.2)) consists of $n+2$ points, then for some subsequence $\left\{n_{k}\right\}$ of the natural numbers, the sets $H_{n_{k}}(f)$ are distributed (in the limit) like the equilibrium distribution of $K$. However, the whole sequence $\left\{H_{n}(f)\right\}_{0}^{\infty}$ need not have this limit distribution, which raises the question of other possible limit distributions (cf. Theorem 6 below).

Finally, our last motivation stems from the "near circularity" property of the image curve $Q_{n}(f, \partial K)$, which was observed and emphasized in several recent papers $[18,19]$. Namely, in some special cases it was observed that for $K=\bar{U}$ the curve $\left\{Q_{n}\left(f, e^{i t}\right): 0 \leq t \leq 2 \pi\right\}$ has winding number $n+1$ and "looks like" a circle. It will follow from our results that this "near circularity" property is very exceptional; that is, for "most" functions this phenomenon does not happen (cf. Theorems 1 and 5). Consequently, we can expect that, in general, the polynomial $C F$ method (cf. [18]) will fail to achieve very good approximation. We will elaborate further on this matter in $\S 3$.

We will present the existence results (except for Theorem 6) in terms of category in the metric space $A(K)$. This allows us to avoid tedious and complicated constructions. Moreover, it carries slightly more information by showing that most of the functions (except for a set of the first category) exhibit the property in question.

This paper is organized as follows. $\S 2$ contains the main results (Theorems $1-6)$, the proofs of which occupy $\S \S 4-9$. In $\S 3$ we mention, mostly without proof, some less immediate consequences.

\section{MAIN RESUlts}

We recall that $K$ always denotes an infinite compact set with connected complement, $K^{0}$ is its interior, and $A(K)$ stands for the set of complex-valued functions $f \in C(K)$ that are analytic on $K^{0}$. Finally, with the best approximating polynomials $P_{n}(f, z) \in \Pi_{n}$ we set

$$
Q_{n}(f, z):=\left(f(z)-P_{n}(f, z)\right) / E_{n}(f), \quad z \in K,
$$

which is the "normalized error" of the $n$th polynomial approximation to $f$.

Our first result asserts that, in general, $\left\{Q_{n}(f, z)\right\}_{n=0}^{\infty}$ tends to zero at no point of $K$.

Theorem 1. The set $S$ of functions $f \in A(K)$ for which

$$
\varlimsup_{n \rightarrow \infty} \min _{z \in K} \frac{\left|f(z)-P_{n}(f, z)\right|}{E_{n}(f)}<1
$$

is of the first category in $A(K)$.

As an immediate consequence we get the following corollary. 
Corollary 1. There exist a function $f \in A(K)$ and a subsequence $\left\{n_{k}\right\}$ of the natural numbers such that

$$
\lim _{k \rightarrow \infty} \frac{\left|f(z)-P_{n_{k}}(f, z)\right|}{E_{n_{k}}(f)}=1
$$

uniformly for $z \in K$.

Theorem 1 and Corollary 1 imply the following somewhat surprising fact: For most (in the sense of category) functions $f \in A(K)$, there exists a subsequence $\left\{n_{k}\right\}$ such that $f(z)-P_{n}(f, z)$ has no zeros in $K$ for $n=n_{1}, n_{2}, \ldots$. Note that this does not contradict the Chebyshev equioscillation theorem for best approximation to a real-valued function on a real interval, which implies that the error must have at least $n+1$ zeros for each $n=0,1,2, \ldots$. Indeed, the collection of real-valued functions on an interval $K$ is only a first category subset of $A(K)$.

As Corollary 1 shows, the best approximants in general do not give better approximation on $K^{0}$ than on $K$. But what about "near best" approximants? The following three results are in this direction.

Theorem 2. Suppose that the complement as well as the interior of $K$ are connected and $f \in A(K)$. Then there are polynomials $p_{n} \in \Pi_{n}, n=1,2, \ldots$, such that

$$
\varlimsup_{n \rightarrow \infty} \frac{\left\|f-p_{n}\right\|_{K}}{E_{n}(f)} \leq 2
$$

and

$$
\varlimsup_{n \rightarrow \infty} \frac{f(z)-p_{n}(z)}{E_{n}(f)}=0
$$

uniformly on compact subsets of $K^{0}$.

We do not know if this result holds when $K^{0}$ is not connected. However, our next result shows that the constant 2 in (2.2) is the best possible.

Theorem 3. Suppose $K$ has nonempty interior. Then there is a function $f \in$ $A(K)$ such that if $p_{n} \in \Pi_{n}, n=1,2, \ldots$, are polynomials with the property

$$
\lim _{n \rightarrow \infty} \frac{f(z)-p_{n}(z)}{E_{n}(f)}=0
$$

uniformly on compact subsets of $K^{0}$, then

$$
\varlimsup_{n \rightarrow \infty} \frac{\left\|f-p_{n}\right\|_{K}}{E_{n}(f)} \geq 2 .
$$

The proof shows that actually for most functions in $A(K)$ (except for those in a set of first category) if (2.4) holds in at least one point of $K^{0}$ for some polynomials $p_{n} \in \Pi_{n}, n=1,2, \ldots$, then (2.5) holds true.

If $K$ has an analytic boundary, then the convergence (2.3) can be replaced by geometric convergence: 
Theorem 4. Suppose that $K$ is bounded by a simple closed analytic curve. Then there is a constant $C$ such that for every $f \in A(K)$ there are polynomials $p_{n} \in \Pi_{n}, n=1,2, \ldots$, satisfying

$$
\left\|f-p_{n}\right\|_{K} \leq C E_{n}(f)
$$

and

$$
\varlimsup_{n \rightarrow \infty}\left(\frac{\left|f(z)-p_{n}(z)\right|}{E_{n}(f)}\right)^{1 / n}<1
$$

uniformly on compact subsets of $K^{0}$.

Our results raise the following bold conjecture that would be the ultimate refinement of Mergelyan's approximation theorem: If $K$ has connected complement and $f \in A(K)$, then there are polynomials $p_{n} \in \Pi_{n}, n=1,2, \ldots$, such that $\left\|f-p_{n}\right\|_{K} \rightarrow 0$ as $n \rightarrow \infty$ and

$$
\varlimsup_{n \rightarrow \infty}\left|f(z)-p_{n}(z)\right|^{1 / n}<1
$$

uniformly on compact subsets of $K^{0}$. This would mean that whenever geometric convergence is permitted, it is actually achieved.

We have already mentioned that $\left\{Q_{n}(f, z)\right\}_{n=0}^{\infty}$ forms a normal family on $K^{0}$ and so we can select convergent subsequences from it. Theorem 1 shows that, in general, there is such a convergent subsequence that converges to a not identically zero function. This raises the question: What possible limits can be obtained from $\left\{Q_{n}(f, z)\right\}_{n=0}^{\infty}$ ? It is easy to see that when $f$ is not a polynomial, no subsequence of $\left\{Q_{n}(f, z)\right\}_{n=0}^{\infty}$ can converge uniformly on $K$. In fact, if we had $\lim _{k \rightarrow \infty} Q_{n_{k}}(f, z)=g(z)$ uniformly, then $g \in A(K)$ and so $\left\|g-P_{0}\right\|_{K}<1$ for some polynomial $P_{0}$. But this would mean

$$
\left\|f-\left(P_{n_{k}}(f)+E_{n_{k}}(f) P_{0}\right)\right\|_{K}<E_{n_{k}}(f),
$$

and at the same time $P_{n_{k}}(f)+E_{n_{k}}(f) P_{0} \in \Pi_{n_{k}}$ for $n_{k}>\operatorname{deg} P_{0}$, which contradicts the fact that we cannot get a better approximation to $f$ from $\Pi_{n_{k}}$ than $E_{n_{k}}(f)$. Thus, the most we can hope for is that we get uniform convergence except for a small neighborhood of a point $z_{0} \in \partial K$. It is also clear that $z_{0}$ cannot be an isolated point of $K$. Now we show that most of the functions $f$ in $A(K)$ are universal in that every $g \in A(K)$ with $\|g\|_{K} \leq 1$ occurs as a limit of a subsequence of $\left\{Q_{n}(f, z)\right\}_{n=0}^{\infty}$ in this sense (since $\left\|Q_{n}(f)\right\|_{K}=1$ for every $n$, other limit functions cannot arise).

To state our result let

$$
U_{\rho}\left(z_{0}\right):=\left\{z:\left|z-z_{0}\right|<\rho\right\}
$$

be the open disk of radius $\rho$ with center at $z_{0}$.

Theorem 5. Let $S_{1}$ be the set of $f \in A(K)$ satisfying the following condition: if $g \in A(K),\|g\|_{K} \leq 1, z_{0} \in \partial K$ is a nonisolated point of $K$, and $\varepsilon, \rho$ are 
arbitrary positive numbers, then there is an $n$ such that

$$
\left|\frac{f(z)-P_{n}(f, z)}{E_{n}(f)}-g(z)\right|<\varepsilon \quad \text { if } z \in K \backslash U_{\rho}\left(z_{0}\right) .
$$

Then $S_{1}$ is a residual set; that is, its complement in $A(K)$ is of the first category.

Corollary 2. There exists $f \in A(K)$ with the property that if $g \in A(K),\|g\|_{K} \leq$ 1 , is arbitrary and $z_{0} \in \partial K$ is a nonisolated point of $K$, then there is a subsequence $\left\{n_{k}\right\}$ of the natural numbers such that

$$
\lim _{k \rightarrow \infty} \frac{f(z)-P_{n_{k}}(f, z)}{E_{n_{k}}(f)}=g(z)
$$

for every $z \in K$, and the convergence is uniform on any closed subset of $K$ not containing $z_{0}$.

Our last result concerns the distribution of the points in the sets

$$
H_{n}=H_{n}(f):=\left\{z \in K:\left|f(z)-P_{n}(f, z)\right|=E_{n}(f)\right\},
$$

called the sets of maximal deviation points of best polynomial approximation. $H_{n}(f)$ must always contain at least $(n+2)$ points and, in general, $H_{n}$ is part of $\partial K$; but it may happen that $H_{n} \equiv \partial K$ for every $n$ (for a nontrivial example see [4]). In [4] it is verified that there is a subsequence $\left\{n_{k}\right\}$ of the natural numbers such that a certain $\left(n_{k}+2\right)$-point subset of $H_{n_{k}}$, called the $\left(n_{k}+2\right)$ Fekete set, has (in the limit) the distribution given by the equilibrium measure of $K$. In [9] an example was given to show that this is not necessarily true for every subsequence $\left\{n_{k}\right\}$. Thus, the question arises as to what possible limit distributions can be achieved this way. To make our question more precise, let us suppose that $H_{n}$ contains exactly $n+2$ points and let

$$
\nu_{n}(f, B):=(1 /(n+2)) \#\left(B \cap H_{n}\right)
$$

be the normalized counting measure for $H_{n}$ on Borel sets $B \subset \mathbf{C}$. The question we address is, what are the possible weak-star limits of different subsequences of $\left\{\nu_{n}(f)\right\}_{n=0}^{\infty}$ ? Obviously, if $\nu_{n_{k}}(f) \rightarrow \nu$ in the weak-star topology of measures on $K$, then $\nu$ must be a probability measure on $\partial K$ vanishing at the set of isolated points of $K$. We show here that for some universal $f \in A(K)$ all such $\nu$ 's can be obtained as weak-star limits of $\left\{\nu_{n}(f)\right\}_{n=0}^{\infty}$.

Theorem 6. There exists an $f \in A(K)$ with the property that whenever $\nu$ is a probability (Borel) measure on $\partial K$ that is zero at the set of isolated points of $K$, then there is a subsequence $\left\{n_{k}\right\}$ of the natural numbers such that the sets $H_{n_{k}}(f)$ contain exactly $n_{k}+2$ points and

$$
\lim _{k \rightarrow \infty} \nu_{n_{k}}(f)=\nu
$$

in the weak-star topology of measures. 


\section{Miscellaneous ConseQuences}

I. Entire functions and singularities on $K$. First of all we mention that the "pathological behavior" of the functions in Theorems 1, 3, 5, and 6 (or in Corollaries 1 and 2) is not due to the existence of a singularity of the function on $\partial K$ or to the lack of it. In fact, we have

Theorem 7. The results of the preceding section are true if the functions (besides belonging to $A(K))$ are assumed to have at least one singularity on $\partial K$. Also, Corollaries 1 and 2 and Theorems 3 and 6 hold for some entire functions $f$.

Proof. Every function analytic on $K$ must belong to at least one of the sets

$$
\begin{aligned}
& S_{m, N}:=\left\{f \in A(K): E_{n}(f) \leq N(1-1 / m)^{n} \text { for every } n \geq 0\right\}, \\
& \qquad m=2,3, \ldots, N=1,2, \ldots .
\end{aligned}
$$

These sets are obviously closed in $A(K)$ and it is easy to see that they have empty interior. Indeed, if this is not the case for some $N, m$, then

$$
E_{n}(F) \leq N_{1}(1-1 / m)^{n}, \quad n=0,1, \ldots,
$$

would hold for some $N_{1}$ and all $F \in A(K),\|F\|_{K} \leq 1$; but this contradicts the fact that for every $n \geq 1$ there is an $F \in A(K),\|F\|_{K} \leq 1$, such that $E_{n}(F)=1$ (see the end of the proof of Theorem 1). Thus we see that the set of functions analytic on $K$ is of the first category in $A(K)$. This proves the first statement of Theorem 7, except for Theorem 6. As for Theorem 6, an easy modification of the proof shows (by choosing $\varepsilon_{k} \rightarrow 0$ suitably) that the function $f$ constructed there has the property $\varlimsup_{n \rightarrow \infty}\left(E_{n}(f)\right)^{1 / n}=1$, and this implies that $f$ has a singularity somewhere on $\partial K$ (cf. $[20, \S \S 4.7,4.9]$ ).

We will not prove the statement concerning entire functions. The proof of Theorem 6 easily yields an entire function $f$ satisfying the stated properties, and similar constructions, based on our proofs below, show that the functions in Corollaries 1 and 2 and in Theorem 3 can also be entire.

II. Best approximants on different sets. To facilitate discussion of best approximation on different sets let us agree to use the symbol of the set as a subscript in $P_{n}(f), E_{n}(f)$, etc. It follows from the Kolmogorov criterion and the proof of Theorem 6 that there exists an entire function $f$ (see also Theorem 7) such that if $z_{0} \in \partial K$ is an arbitrary nonisolated point and $\delta>0$, then there are infinitely many $n$ 's for which the best approximation of $f$ out of $\Pi_{n}$ taken on $K$ coincides with that on $K_{1}:=K \cap \overline{U_{\delta}\left(z_{0}\right)}$ (i.e., $\left.P_{n}(f)_{K} \equiv P_{n}(f)_{K_{1}}\right)$ and the error of approximation is also the same (i.e., $\left.E_{n}(f)_{K}=E_{n}(f)_{K_{1}}\right)$. We have already mentioned that every point on $\partial K_{1}$, except for points in a set of zero capacity, is a limit point of the extreme point sets $\left\{H_{n}(f)_{K_{1}}\right\}_{n=1}^{\infty}$. Thus, if $\partial U_{\delta}\left(z_{0}\right) \cap K^{0} \neq \varnothing$ (and $f$ is not a polynomial), then $P_{n}(f)_{K} \equiv P_{n}(f)_{K_{1}}$ and $E_{n}(f)_{K}=E_{n}(f)_{K_{1}}$ cannot happen for all large $n$.

Note also that if we drop the condition $E_{n}(f)_{K}=E_{n}(f)_{K_{1}}$, then it may happen that $P_{n}(f)_{K} \equiv P_{n}(f)_{K_{1}}$ for all $n$ for two different sets $K$ and $K_{1}$ with 
$K_{1} \subseteq K^{0}$. In fact, it is easy to see (by looking at the real parts, for example) that for any numbers $0<a_{k}<1 /\left[(2 k)^{k}\right]$ ! the function

$$
f(z)=\sum_{k=1}^{\infty} a_{k} z^{(2 k+1) ! !}, \quad(2 k+1) ! !:=(2 k+1)(2 k-1) \cdots 3 \cdot 1,
$$

has the partial sums of the defining series as best polynomial approximants on any disk with center at the origin (see also [1]).

An easy modification of the proof of Theorem 6 also shows that there are pairwise disjoint compact sets (say $\left.\overline{U \cap U_{\delta}\left(z_{j}\right)}, 1 \leq j \leq N\right) K_{1}, K_{2}, \ldots, K_{N}$ and an entire function $f$ such that

$$
P_{n}(f)_{K_{j}} \equiv P_{n}(f)_{K_{i}}, \quad 0 \neq E_{n}(f)_{K_{j}}=E_{n}(f)_{K_{i}}, 1 \leq i, j \leq N,
$$

occurs for infinitely many $n$.

III. Zeros of best approximating polynomials and analytic continuation. It was shown in [3] that if $f \in A(K)$ is not analytic on $K$, then every point on $\partial K$, except perhaps points in a set of zero capacity, is a limit point of the zeros of $\left\{P_{n}(f)\right\}_{n=0}^{\infty}$. Replacing $K$ by $K_{1}=\overline{K \cap U_{\delta}\left(z_{0}\right)}$ (see $\S$ II above) we can see from Theorem 5 (see also Theorem 7) that, on the other hand, it may happen that we get a large zero-free region, namely $K$, for $P_{n}(f)_{K_{1}}$ for infinitely many $n$.

The accumulation of zeros just mentioned rules out the possibility of analytic continuation by best polynomial approximants if we use all the terms in the sequence $\left\{P_{n}(f)_{K_{1}}\right\}_{n=0}^{\infty}$ (cf. [13]). On the other hand, as we have just seen, certain subsequences of $\left\{P_{n}(f)_{K_{1}}\right\}_{n=0}^{\infty}$ can provide analytic continuation to a large region, namely $K$.

IV. "Near circularity". Let $K=\bar{U}$ be the closed unit disk. It can easily be seen from Theorem 1 or Corollary 1 that for most functions $f$ the curves $\gamma_{n}:=\left(f-P_{n}(f)\right)\left(e^{i t}\right), 0 \leq t \leq 2 \pi$, have winding number 0 (with respect to the origin) for infinitely many $n$. Thus, the near circularity property of [18] does not hold for most of the functions in $A(K)$ (not even for entire functions). The curve $\gamma_{n}$ is also far from "looking like" a circle as is shown by Theorem 5.

V. Carathéodory-Fejér approximation. We have already mentioned that an approximation method based on the near circularity property was introduced by Trefethen [18]. This so-called $C F$ method is the following. Let $f$ be in the disk algebra $\mathscr{A}$ with Taylor series $f(z)=\sum_{k=0}^{\infty} a_{k} z^{k}$. The problem of best polynomial approximation to $f$ out of $\Pi_{n}$ is then equivalent to minimizing

$$
\left\|\sum_{k=0}^{n} c_{k} z^{k}+\sum_{k=n+1}^{\infty} a_{k} z^{k}\right\|_{\omega}
$$

over all $(n+1)$-tuples $\left(c_{0}, \ldots, c_{n}\right)$. The $\mathrm{CF}$ method starts with truncating the Taylor series at some $k=N>n$ so that $\sum_{k=N+1}^{\infty} a_{k} z^{k}$ is negligible and setting 
$p(z):=\sum_{k=0}^{N-n-1} a_{N-k} z^{k}$. By a result of Carathéodory and Fejér, there is a unique power series extension

$$
B(z)=p(z)+\sum_{k=N-n}^{\infty} c_{k}^{*} z^{k}
$$

analytic in the unit disk that minimizes $\|B\|_{H^{\infty}}$ among all such extensions. The minimizing $B$ turns out to be a finite Blaschke product with at most $N-n-$ 1 zeros, and $B$ can be found by solving a certain eigenvalue problem for a Hankel matrix formed from the coefficients of $p$. Finally, we take $p_{n, N}(z):=$ $\sum_{k=0}^{n}\left(a_{k}-c_{N-k}^{*}\right) z^{k}$ as the CF approximant of $f$.

The above method has not been specified completely since it must be preceded by a truncation that leaves the remainder term "negligible." In the literature, the place $N$ where truncation is suggested for the $n$th approximant is some fixed number (say $2 n+2$ ) depending on $n$. However, it turns out that no fixed sequence can serve as good truncation places; what is more, all the truncations made sufficiently far can be uniformly bad. In fact, it can be proved that if $c>1$, there exists a function $f \in \mathscr{A}$ for which

$$
\varlimsup_{n \rightarrow \infty} \inf _{N \geq c n} \frac{\left\|f-p_{n, N}\right\|_{\mathscr{A}}}{E_{n}(f) \log n}>0 .
$$

Thus, in general the CF approximants give only the rate of approximation $\left\{E_{n}(f) \log n\right\}$, which is the same as that given by the partial sums of the Taylor series. There are functions $f \in \mathscr{A}$ for which the CF approximants diverge and they are in general not better than the partial sums, even for entire functions.

Of course, it may happen that for some special classes of functions (say with rapidly decreasing Taylor coefficients), the CF method provides sharp estimates. However, we wish to emphasize that the polynomial Carathéodory-Fejér method is not truly effective for a large class of functions. This topic will be treated in more detail in a forthcoming paper of the authors [14].

\section{Proof of Theorem 1}

Lemma 1. Let $K$ be a compact set with connected complement. Then there exists a subset $\widetilde{K}$ of $\partial K$ such that $\widetilde{K}$ is dense in $\partial K$, and if $z_{1}, \ldots, z_{n} \in \widetilde{K}$ are arbitrary distinct points and $\xi_{1}, \ldots, \xi_{n}$ are arbitrary numbers of modulus 1 , then for every $\varepsilon>0$ there is a $g \in A(K)$ such that $\|g\|_{K}=1, g\left(z_{j}\right)=\xi_{j}$, $1 \leq j \leq n$, and

$$
\min _{z \in K}|g(z)| \geq 1-\varepsilon .
$$

Proof. Let $\widetilde{K}$ be the subset of $\partial K$ that consists of all the points accessible from the complement $G:=\overline{\mathbf{C}} \backslash K$ of $K$ through Jordan curves, i.e., the set of points $z \in \partial K$ which are the endpoints of Jordan curves contained in $G \cup\{z\}$. It is easy to see that $\widetilde{K}$ is dense in $\partial K$. In fact, if $z \in \partial K$ arbitrary and $w \in G$ is close to $z$, then the closest point of $\{w+t(z-w): 0 \leq t \leq 1\} \cap K$ to $w$ is clearly accessibie and is close to $z$. 
Now let $z_{1}, \ldots, z_{n} \in \widetilde{K}$ be $n$ points. Then there are Jordan curves $\gamma_{j} \subseteq$ $G \cup\left\{z_{j}\right\}$ with endpoint at $z_{j}, 1 \leq j \leq n$. Since $G$ is open, it is possible to construct (close to $\gamma_{j}$ ) another Jordan curve $\gamma_{n+j}$ contained in $G \cup\left\{z_{j}\right\}$ such that $\gamma_{n+j}$ has endpoint at $z_{j}$ but otherwise $\gamma_{j}$ and $\gamma_{n+j}$ are disjoint, i.e., $\gamma_{j} \cap \gamma_{n+j}=\left\{z_{j}\right\}$. Without loss of generality we may assume that each $\gamma_{j}$, $1 \leq j \leq 2 n$, has one endpoint on a circle $C$ containing $K$ in its interior and that the $\gamma_{j}$ 's are disjoint in $G$; i.e., if $\gamma_{j} \cap \gamma_{l} \neq \varnothing, l>j$, then $l=n+j$ and $\gamma_{j} \cap \gamma_{l}=\left\{z_{j}\right\}$ (extend and alter the curves $\gamma_{j}$ if necessary).

The connected component $G_{0}$ of $\mathbf{C} \backslash\left(C \cup\left(\bigcup_{j=1}^{2 n} \gamma_{j}\right)\right)$ that contains $K \backslash\left\{z_{1}, \ldots, z_{n}\right\}$ is bounded by the Jordan arcs $\gamma_{j}, 1 \leq j \leq 2 n$, and some arcs of $C$ (see Figure 1); hence $G_{0}$ is a Jordan domain. Thus there is a conformal mapping $\phi_{0}$ of $G_{0}$ onto the unit disk $U$ that is continuous and one-to-one between $\partial G_{0}$ and $\partial U$ (see [10, Theorem 2.24]). Notice that $\phi_{0}$ carries $z_{1}, \ldots, z_{n}$ into $\partial U$ but every other point of $K$ is mapped into $U$.

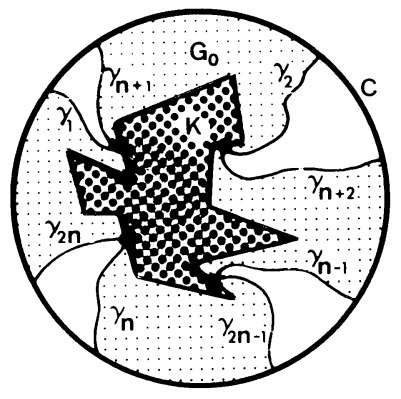

FIGURE 1

Let $\phi_{1}$ be a conformal mapping of $U$ onto a Jordan domain $D$ contained in $\{z: 1-\varepsilon \leq|z| \leq 1\}$ that contains $\xi_{1}, \ldots, \xi_{n}$ on its boundary (see Figure $2)$. Finally, by the Rudin theorem [6, p. 81] there is a function $h$ in the disk algebra $\mathscr{A}=A(\bar{U})$ such that $\|h\|_{\mathscr{A}} \leq 1$ and $h$ takes the value $\phi_{1}^{-1}\left(\xi_{j}\right)$ at the point $\phi_{0}\left(z_{j}\right)$ for $1 \leq j \leq n$. Then $g(z):=\phi_{1} \circ h \circ \phi_{0}(z)$ maps $K$ into $\bar{D}$ in such a way that $z_{j}$ is mapped into $\xi_{j}$ for every $1 \leq j \leq n$, and this proves the lemma.

Proof of Theorem 1. Let

$$
S_{m, N}:=\left\{f \in A(K): \min _{z \in K}\left|Q_{n}(f, z)\right| \leq 1-\frac{1}{m} \text { for } n \geq N\right\} .
$$

Then $S=\bigcup_{m=1}^{\infty} \bigcup_{N=1}^{\infty} S_{m, N}$; hence if $S$ is not of the first category, then for some $m$ and $N$ the set $S_{m, N}$ is not nowhere dense in $A(K)$. It easily follows from the strong continuity of the operator of best $n$th polynomial approximation that $S_{m, N}$ is a closed subset of $A(K)$. In fact, if $\left\{f_{\nu}\right\}_{\nu=1}^{\infty} \subseteq S_{m, N}$ and 

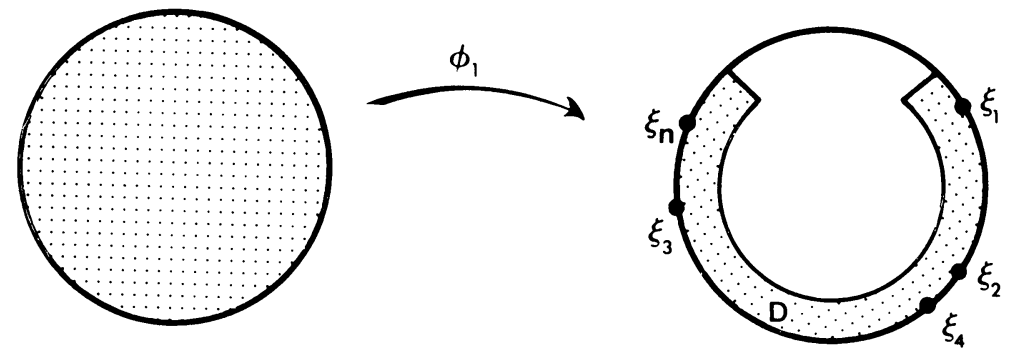

FIGURE 2

$f_{\nu}$ uniformly converges to $f$ on $K$, then for every $n \geq N$ there are two possibilities:

(i) $f$ is a polynomial of degree at most $n$, in which case $Q_{n}(f, z) \equiv 0$ and $f \in S_{m, N}$, or

(ii) $E_{n}(f) \neq 0$. In this case $E_{n}\left(f_{\nu}\right) \rightarrow E_{n}(f)$ and $P_{n}\left(f_{\nu}, z\right) \rightarrow P_{n}(f, z)$ uniformly in $z \in K$ as $\nu \rightarrow \infty$, which imply the uniform convergence of $Q_{n}\left(f_{\nu}, z\right)$, and so

$$
\min _{z \in K}\left|Q_{n}(f, z)\right| \leq \varlimsup_{\nu \rightarrow \infty} \min _{z \in K}\left|Q_{n}\left(f_{\nu}, z\right)\right| \leq 1-\frac{1}{m},
$$

i.e., $f \in S_{m, N}$.

Since $S_{m, N}$ is closed and not nowhere dense in $A(K)$, there is an $f_{0} \in A(K)$ and a $\delta_{0}>0$ such that the $\delta_{0}$-neighborhood of $f_{0}$ in $A(K)$ is contained in $S_{m, N}$. Choose a polynomial $P_{0}$ with $\left\|f_{0}-P_{0}\right\|<\delta_{0} / 2$, and set $n_{0}:=$ $\max \left(N, \operatorname{deg} P_{0}\right)$. If $f(\not \equiv 0)$ is any function in $A(K)$, then the function

$$
f^{*}(z):=P_{0}(z)+\left(\delta_{0} / 2\right)\|f\|_{K}^{-1} f(z)
$$

belongs to the $\delta_{0}$-neighborhood of $f_{0}$; hence

$$
\min _{z \in K}\left|Q_{n_{0}}\left(f^{*}, z\right)\right| \leq 1-\frac{1}{m} .
$$

But $Q_{n_{0}}\left(f^{*}, z\right) \equiv Q_{n_{0}}(f, z)$ and we can conclude that for every function $f \in$ $A(K)$

$$
\min _{z \in K}\left|Q_{n_{0}}(f, z)\right| \leq 1-\frac{1}{m} .
$$

That this is impossible can be seen as follows.

By Lemma 1 , if $z_{1}, \ldots, z_{n_{0}+2} \in \widetilde{K}$ and $\xi_{1}, \ldots, \xi_{n_{0}+2},\left|\xi_{j}\right|=1$, are arbitrary numbers, then there is an $f \in A(K)$ such that $\|f\|_{K}=1, f\left(z_{j}\right)=\xi_{j}, 1 \leq j \leq$ $n_{0}+2$, and

$$
\min _{z \in K}|f(z)|>1-\frac{1}{m}
$$


We claim that for fixed $z_{1}, \ldots, z_{n_{0}+2}$ we can choose $\xi_{1}, \ldots, \xi_{n_{0}+2}$ in such a way that 0 is the best approximant of $f$ out of $\Pi_{n_{0}}$. In that case we will have $Q_{n_{0}}(f ; z) \equiv f(z)$ and so $(4.2)$ will contradict (4.1).

In order to prove the claim consider the set

$$
\pi_{n_{0}}:=\left\{\left(P\left(z_{1}\right), \ldots, P\left(z_{n_{0}+2}\right)\right): P \in \Pi_{n_{0}}\right\}
$$

in the complex $l_{\infty}^{n_{0}+2}$ space. Then $\pi_{n_{0}}$ is an $\left(n_{0}+1\right)$-dimensional subspace in $l_{\infty}^{n_{0}+2}$; hence, by a result of M. G. Krein (see [11, p. 12]) there is a $\xi \in l_{\infty}^{n_{0}+2}$ of norm 1 such that $\mathbf{0}$ is the best approximant of $\boldsymbol{\xi}$ out of $\pi_{n_{0}}$. Clearly, each coordinate of $\boldsymbol{\xi}$ must be of absolute value one. Thus, if $\boldsymbol{\xi}=\left(\xi_{1}, \ldots, \xi_{n_{0}+2}\right)$ and $f$ is the above function constructed to $\xi_{1}, \ldots, \xi_{n_{0}+2}$, then no polynomial can approximate $f$ on the set $\left\{z_{1}, \ldots, z_{n_{0}+2}\right\}$ better than the zero polynomial and so $P_{n_{0}}(f, z) \equiv 0$, as we claimed.

Because of its important role in this and later proofs of the paper, we mention that the vector $\boldsymbol{\xi}=\left(\xi_{1}, \ldots, \xi_{n_{0}+2}\right)$ that yields $P_{n_{0}}(f, z) \equiv 0$ can be determined explicitly. Indeed, if

$$
w(z):=\prod_{k=1}^{n_{0}+2}\left(z-z_{k}\right),
$$

we can put

$$
\xi_{j}=\exp \left(i \arg w^{\prime}\left(z_{j}\right)\right), \quad 1 \leq j \leq n_{0}+2 .
$$

Then, with $f\left(z_{j}\right)=\xi_{j}$, we have for any $q \in \Pi_{n_{0}}$,

$$
\begin{aligned}
\sum_{j=1}^{n_{0}+2} \frac{1}{\left|w^{\prime}\left(z_{j}\right)\right|} \overline{f\left(z_{j}\right)} q\left(z_{j}\right) & =\sum_{j=1}^{n_{0}+2} \frac{\overline{\xi_{j}} q\left(z_{j}\right)}{\left|w^{\prime}\left(z_{j}\right)\right|}=\sum_{j=1}^{n_{0}+2} \frac{q\left(z_{j}\right)}{w^{\prime}\left(z_{j}\right)} \\
& =q\left[z_{1}, \ldots, z_{n_{0}+2}\right]=0,
\end{aligned}
$$

and this discrete orthogonality together with the fact that $\|f\|_{K}=1$ implies $P_{n_{0}}(f, z) \equiv 0$ (cf. [16, p. 14]).

\section{Proof of Theorem 2}

Let $D$ be an open disk containing $K$ and for $m>0$ let $z_{1}, \ldots, z_{s} \in D \backslash K$ be finitely many points such that to every $z \in D \backslash K$ there is at least one $z_{j}$ closer than $1 / \mathrm{m}$. Since the complement of $K$ is connected, it is easy to see (say, by induction; that we can connect the points $z_{j}$ to one another or to $\partial D$ by broken lines in such a way that the complement of this broken line system in $D$ is simply connected and contains $K$. In other words, there is a closed set $S_{m} \subseteq \bar{D} \backslash K$ such that $G_{m}:=D \backslash S_{m}$ is a simply connected domain and for every $z \in D \backslash K$ we have $\operatorname{dist}\left(z, S_{m}\right)<1 / m$. This implies that if $\mathscr{N}$ is any subsequence of the natural numbers, then the set

$$
\left\{z: \exists \rho, m_{z} \text { such that } U_{\rho}(z) \subseteq G_{m}, m \in \mathcal{N}, m \geq m_{z}\right\}
$$


is exactly $K^{0}$; that is, $\left\{G_{m}\right\}$ converges to $K^{0}$ in Carathéodory's sense (cf. [10, $\S 4])$.

Let $z_{0} \in K^{0}$ be arbitrary but fixed and let $\varphi_{m}, \varphi$ denote the conformal mappings of $G_{m}$ and $K^{0}$, respectively, onto $U$ normalized by

$$
\begin{aligned}
\varphi_{m}\left(z_{0}\right) & =0, & & \varphi_{m}^{\prime}\left(z_{0}\right)>0, \\
\varphi\left(z_{0}\right) & =0, & & \varphi^{\prime}\left(z_{0}\right)>0 .
\end{aligned}
$$

By Carathéodory's theorem [10, Theorem 2.1] $\varphi_{m} \rightarrow \varphi$ uniformly on compact subsets of $K^{0}$ and $\varphi_{m}^{-1} \rightarrow \varphi^{-1}$ uniformly on compact subsets of $U$ as $m \rightarrow \infty$.

If $1>\eta>0$ is given, let $K^{\eta}$ be a compact subset of $K^{0}$ such that the image of $K^{\eta}$ under the mapping $\varphi$ contains the disk

$$
U_{1-\eta / 4}:=\{z:|z|<1-\eta / 4\} .
$$

Then, for $m \geq m(\eta)$, the image of $K^{\eta}$ under the mapping $\varphi_{m}$ contains $U_{1-\eta / 2}$. Since $K$ is a compact subset of $G_{m(\eta)}$, we can uniformly approximate $\varphi_{m(\eta)}$ on $K$ by polynomials, and let $P_{\eta}$ be such a good polynomial approximant of $\varphi_{m(\eta)}$. We can ensure that $P_{\eta}$ is one-to-one on $K$, for $\varphi_{m(\eta)}$ is one-toone on $G_{m(\eta)}$ and so, by Rouché's theorem, every good approximant of $\varphi_{m(\eta)}$ will inherit this property on $K$ (more precisely, to achieve this we have to approximate $\varphi_{m(\eta)}$ on a larger compact subset of $G_{m(\eta)}$ containing $K$ in its interior, but this is always possible). Furthermore, we can assume that the approximant $P_{\eta}$ is so good that, under the mapping $P_{\eta}$, the image of $K^{\eta}$ contains $U_{1-\eta}$, the image of $K$ is contained in $U$, and the inverse image of $U_{1-\eta^{1 / 4}}$ contains $\varphi^{-1}\left(U_{1-2 \eta^{1 / 4}}\right)$.

Let

$$
F(w)=F_{\eta, n}(w):=\frac{f-P_{n}(f)}{E_{n}(f)} \circ P_{\eta}^{-1}(w)=Q_{n}(f) \circ P_{\eta}^{-1}(w)
$$

with $w \in U_{1-\eta}$, and let

$$
F(w)=a_{0}+a_{1} w+a_{2} w^{2}+\cdots
$$

be its Taylor expansion around $w=0$ with partial sums denoted by $s_{m}(w)$. For $M<N$ we set

$$
\begin{aligned}
\tau_{M, N}(w)=\tau_{M, N}(F, w) & :=\sum_{k=0}^{M-1} a_{k} w^{k}+\sum_{k=M}^{N-1} \frac{N-k}{N-M} a_{k} w^{k} \\
& =\frac{1}{N-M}\left(s_{M}(w)+\cdots+s_{N-1}(w)\right) \\
& =\frac{1}{N-M}\left(N \sigma_{N-1}(w)-M \sigma_{M-1}(w)\right),
\end{aligned}
$$

where $\sigma_{k}(w)$ denote the $(C, 1)$ means of the Taylor expansion (5.1). It is well known $($ see $[17, \S 8.7 .1])$ that

$$
\left\|\sigma_{k}\right\|_{\partial U_{1-\eta}} \leq\|F\|_{\partial U_{1-\eta}} \leq 1
$$


for all $k$, and so

$$
\left\|\tau_{M, N}\right\|_{\partial U_{1-\eta}} \leq(N+M) /(N-M) \text {. }
$$

Hence, since $\tau_{M, N}$ is a polynomial of degree at most $N$, we get from Bernstein's inequality (see $[20, \S 4.6])$

$$
\left\|\tau_{M, N}\right\|_{\partial U} \leq \frac{N+M}{N-M}(1-\eta)^{-N}
$$

Finally, making use of the fact that the first $M$ Taylor coefficients of $F-\tau_{M, N}$ vanish, we deduce from Schwarz's lemma the estimate (see also (5.2))

$$
\begin{aligned}
\left|\frac{f(z)-P_{n}(f, z)}{E_{n}(f)}-\tau_{M, N} \circ P_{\eta}(z)\right| & =\left|F(w)-\tau_{M, N}(w)\right| \\
& \leq 2 \frac{N+M}{N-M}\left(\frac{1-\rho}{1-\eta}\right)^{M}
\end{aligned}
$$

where $w=P_{\eta}(z)$ and $z \in \varphi^{-1}\left(U_{1-2 \rho}\right) \subseteq P_{\eta}^{-1}\left(U_{1-\rho}\right), \rho=\eta^{1 / 4}$.

Let now $\rho=1 / k, \eta=1 / k^{4}, M=k^{2}$, and $N=k^{3}$. The above construction shows that the polynomial $R_{k, n}(f, z):=E_{n}(f) \tau_{M, N} \circ P_{\eta}(z)$ has degree at most $\left(\operatorname{deg} P_{k^{-4}}\right) \cdot\left(k^{3}-1\right)=: n(k)$. Furthermore,

$$
\left\|R_{k}(f, z)\right\|_{K} \leq E_{n}(f)\left(1-\frac{1}{k^{4}}\right)^{-k^{3}} \frac{k^{3}+k^{2}}{k^{3}-k^{2}}=E_{n}(f)\left(1+O\left(\frac{1}{k}\right)\right)
$$

and on $\varphi^{-1}\left(U_{1-2 / k}\right)$ the function $R_{k}(f, z)$ approximates $f-P_{n}(f)$ with error at most $4 E_{n}(f) e^{-k}(k \geq 5)$. Without loss of generality we can assume $n(k)<$ $n(k+1)$ for every $k$. Thus, by choosing

$$
p_{n}(z):=P_{n}(f, z)+R_{k, n}(f, z)
$$

for $n(k) \leq n<n(k+1), k=1,2, \ldots$, we get the required polynomial sequence since $\left\{\varphi^{-1}\left(U_{1-2 / k}\right)\right\}_{k=3}^{\infty}$ is an increasing sequence of domains converging to $K^{0}$.

\section{Proof of Theorem 3}

The proof runs parallel with that of Theorem 1; therefore, we omit some details.

Let $z_{0} \in K^{0}$ be an arbitrary fixed point and consider the set

$$
\begin{aligned}
& S_{m, N}:=\left\{f \in A(K): \text { for } n \geq N \text { there is a } p_{n} \in \Pi_{n}\right. \text { such that } \\
& \left.\qquad \frac{\left\|f-p_{n}\right\|_{K}}{E_{n}(f)} \leq 2-\frac{1}{m}, \frac{\left|f\left(z_{0}\right)-p_{n}\left(z_{0}\right)\right|}{E_{n}(f)} \leq \frac{1}{16 m}\right\} .
\end{aligned}
$$

If the conclusion of Theorem 3 is false, then $\bigcup_{m, N} S_{m, N}=A(K)$. Any (on $K$ ) bounded subset of $\Pi_{n}$ (with fixed $n$ ) is compact and this easily implies that 
each $S_{m, N}$ is a closed set in $A(K)$ (see also the proof of Theorem 1). Thus, exactly as in the proof of Theorem 1, we get that some $S_{m, N}$ must contain an $f_{0}$ together with a $\delta_{0}$ neighborhood of it. Let $P_{0}$ be a polynomial of degree $n_{0}>N$ approximating $f_{0}$ with error $\delta_{0} / 2$. If $f \in A(K)$ is an arbitrary nonidentically zero function, then the function

$$
f^{*}(z):=P_{0}(z)+\left(\delta_{0} / 2\right) f(z) /\|f\|_{K}
$$

belongs to the $\delta_{0}$ neighborhood of $f_{0}$ and so to $S_{m, N}$. This means that there is a polynomial $P_{n_{0}}^{*} \in \Pi_{n_{0}}$ with the properties

$$
\frac{\left\|f^{*}-P_{n_{0}}^{*}\right\|_{K}}{E_{n_{0}}(f)} \leq 2-\frac{1}{m}, \quad \frac{\left|f^{*}\left(z_{0}\right)-P_{n_{0}}^{*}\left(z_{0}\right)\right|}{E_{n_{0}}(f)} \leq \frac{1}{16 m} .
$$

But then, for

$$
P_{n_{0}}:=\left(\delta_{0} / 2\right)^{-1}\|f\|_{K}\left(P_{n_{0}}^{*}-P_{0}\right)
$$

we have

$$
\left(f-P_{n_{0}}\right)(z) / E_{n_{0}}(f) \equiv\left(f^{*}-P_{n_{0}}^{*}\right)(z) / E_{n_{0}}\left(f^{*}\right),
$$

and we can conclude that for every $f \in A(K)$ there is a $P_{n_{0}} \in \Pi_{n_{0}}$ such that

$$
\frac{\left\|f-P_{n_{0}}\right\|_{K}}{E_{n_{0}}(f)} \leq 2-\frac{1}{m}, \quad \frac{\left|f\left(z_{0}\right)-P_{n_{0}}\left(z_{0}\right)\right|}{E_{n_{0}}(f)} \leq \frac{1}{16 m} .
$$

Below we show that this is not the case and this contradiction will prove Theorem 3.

Since $n_{0}$ is fixed, the polynomials in $\Pi_{n_{0}}$ having sup norm (on $K$ ) at most equal to 3 are equicontinuous on $K$. Let us now consider the subset $\widetilde{K}$ of $\partial K$ mentioned in Lemma 1 and let $K_{0}$ be the component of $K^{0}$ that contains $z_{0}$. If $P \in \Pi_{n_{0}},\|P\|_{K} \leq 3$, and $\left|P\left(z_{0}\right)\right| \geq 1-1 / 8 m$, then there is a $z_{0}^{*} \in$ $\partial K_{0}$ with $\left|P\left(z_{0}^{*}\right)\right| \geq 1-1 / 8 m$. Furthermore, using the equicontinuity just mentioned, $\left|P\left(z_{0}^{*}\right)-P(z)\right| \leq 1 / 8 m$ holds in a $\delta$-neighborhood of $z_{0}^{*}$, where $\delta$ is independent of $P$ and $z_{0}^{*} \in \partial K_{0}$. Since $\widetilde{K}$ is dense in $\partial K$ and $\partial K_{0}$ does not contain isolated points (the complement of $K$ is assumed to be connected), there are $30 m$ disjoint finite subsets $\widetilde{K}_{1}, \ldots, \widetilde{K}_{30 m}$ of $\widetilde{K}$ such that every $\delta$ neighborhood of every $z^{*} \in \partial K_{0}$ meets each of the $\widetilde{K}_{j}$ 's. Let $z_{1}, \ldots, z_{n_{0}+2}$ be $n_{0}+2$ additional points from $\widetilde{K}$. We define the function

$$
\xi: \bigcup_{j=1}^{30 m} \widetilde{K}_{j} \cup\left\{z_{1}, \ldots, z_{n_{0}+2}\right\} \rightarrow \partial U
$$

by

$$
\xi(z):=e^{i j 2 \pi / 30 m} \text { if } z \in \widetilde{K}_{j}, 1 \leq j \leq 30 m,
$$

and on $\left\{z_{1}, \ldots, z_{n_{0}+2}\right\}$ we define $\xi$ in such a way that if $f \in A(K),\|f\|_{K} \leq 1$, agrees with $\xi$ on $\left\{z_{1}, \ldots, z_{n_{0}+2}\right\}$, then zero is its best approximant out of $\Pi_{n_{0}}$ 
(see the proof of Theorem 1). By Lemma 1, there is an $f \in A(K)$ that is an extension of $\xi$ and has the properties $\|f\|_{K}=1$,

$$
\min _{z \in K}|f(z)| \geq 1-\frac{1}{16 m} .
$$

Then $E_{n_{0}}(f)=1$ and we claim that for this $f$ there is no polynomial in $\Pi_{n_{0}}$ that can satisfy $(6.1)$.

In fact, suppose $P_{n_{0}} \in \Pi_{n_{0}}$ satisfies $\left|f\left(z_{0}\right)-P_{n_{0}}\left(z_{0}\right)\right| \leq 1 / 16 m$. Then $\left|P_{n_{0}}\left(z_{0}\right)\right| \geq 1-1 / 8 m$ and so there is a $z_{0}^{*} \in \partial K_{0}$ for which $\left|P_{n_{0}}\left(z_{0}^{*}\right)\right| \geq 1-1 / 8 m$. If $\left\|P_{n_{0}}\right\|_{K}>3$, then $\left\|f-P_{n_{0}}\right\|_{K}>2$, so we may assume $\left\|P_{n_{0}}\right\|_{K} \leq 3$. By our construction above every $\widetilde{K}_{j}$ meets the $\delta$-neighborhood of $z_{0}^{*}$; that is, for every $j$ there is a $z_{j}^{*} \in \widetilde{K}_{j}$ such that

$$
\left|P_{n_{0}}\left(z_{0}^{*}\right)-P_{n_{0}}\left(z_{j}^{*}\right)\right| \leq 1 / 8 m
$$

But $f\left(z_{j}^{*}\right)=\exp (i j 2 \pi / 30 m)$, and we get from the previous inequality that for some $j$,

$$
\begin{aligned}
\left|f\left(z_{j}^{*}\right)-P_{n_{0}}\left(z_{j}^{*}\right)\right| & \geq\left|f\left(z_{j}^{*}\right)-P_{n_{0}}\left(z_{0}^{*}\right)\right|-1 / 8 m \\
& \geq 1-2 \pi / 30 m+\left|P_{n_{0}}\left(z_{0}^{*}\right)\right|-1 / 8 m \\
& \geq 1-1 / 4 m+1-1 / 8 m-1 / 8 m=2-1 / 2 m,
\end{aligned}
$$

which means that $P_{n_{0}}$ does not satisfy $(6.1)$ as we claimed above. This completes the proof.

\section{Proof of Theorem 4}

We use the de la Vallée Poussin means (see the proof of Theorem 2) applied to the Faber expansion of $\left(f-P_{n}(f)\right) / E_{n}(f)$.

Our assumption is that $K$ is bounded by a simple closed analytic curve $\gamma$. Then the complement $G$ of $K$ in $\overline{\mathbf{C}}$ can be mapped conformally onto the exterior of a circle $C_{R}=\{z:|z|=R\}$ by a function $\phi$ normalized by $\phi(\infty)=\infty, \lim _{z \rightarrow \infty} \phi(z) / z=1$ (see [10, §14]). If

$$
\phi(z)=z+\alpha_{0}+\alpha_{-1} / z+\cdots
$$

is the Laurent expansion of $\phi$ at infinity, then the expansion of $\phi^{n}$ is

$$
\phi^{n}(z)=z^{n}+\alpha_{n-1}^{(n)} z^{n-1}+\cdots+\alpha_{0}^{(n)}+\alpha_{-1}^{(n)} / z+\cdots .
$$

The polynomials

$$
F_{n}(z):=z^{n}+\alpha_{n-1}^{(n)} z^{n-1}+\cdots+\alpha_{0}^{(n)}
$$

are called the Faber polynomials of $K$.

Since $\gamma$ is analytic, there is an $r<R$ such that $\phi$ can be extended to a conformal mapping of the unbounded component of the complement of a curve $\gamma_{r} \subseteq K^{0}$ onto the exterior of the circle $C_{r}$ (see Figure 3) (cf. [5, p. 45]). Clearly, if for $r \leq \rho \leq R, K_{\rho}$ denotes the compact set bounded by the curve $\gamma_{\rho}=\phi^{-1}\left(C_{\rho}\right)$, then the Faber polynomials of $K_{R}=K$ and $K_{\rho}$ are identical (in 


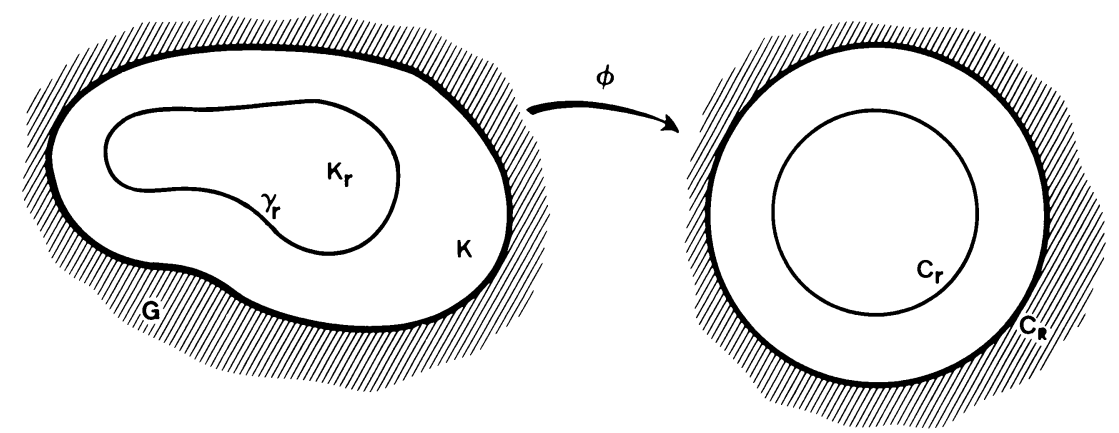

Figure 3

what follows we may assume $r<R$ so large that $\gamma_{\rho}$ is a simple closed analytic curve for $r \leq \rho \leq R$ ).

Consider now an arbitrary function $H$ from $A\left(\bar{U}_{R}\right)$ and let

$$
c_{k}(H):=\frac{1}{2 \pi i} \int_{C_{R}} \frac{H(\xi)}{\xi^{k+1}} d \xi
$$

be its Taylor coefficients. With

$$
t_{n, k}:= \begin{cases}1 & \text { if } k \leq[n / 2], \\ \frac{n-k}{n-[n / 2]} & \text { if }[n / 2]<k \leq n,\end{cases}
$$

we form the de la Vallee Poussin means of the Taylor expansion of $H$ :

$$
\tau_{n}(H, w):=\sum_{k=0}^{n} t_{n, k} c_{k}(H) w^{k} .
$$

Then $\left\|\tau_{n}(H)\right\|_{\bar{U}_{R}} \leq 3\|H\|_{\bar{U}_{R}}$ (see the proof of Theorem 2). Since the first [n/2] terms are missing from the Taylor expansion of $H-\tau_{n}(H)$, Schwarz's lemma gives, for $|w|<R$,

$$
\left|H(w)-\tau_{n}(H, w)\right| \leq 4(|w| / R)^{n / 2}\|H\|_{\bar{U}_{R}} .
$$

In other words, if $I$ is the identity operator and $\tau_{n}$ is the operator that associates $\tau_{n}(H)$ with $H$, then for $\rho<R$

$$
\left\|I-\tau_{n}\right\|_{A\left(\bar{U}_{R}\right) \rightarrow A\left(\bar{U}_{\rho}\right)} \leq 4(\rho / R)^{n / 2} .
$$

Now consider for $r \leq \rho \leq R$ the mapping $T_{\rho}$ from $A\left(K_{\rho}\right)$ onto $A\left(\bar{U}_{\rho}\right)$ defined for polynomials by

$$
T_{\rho}: a_{0}+a_{1} w+\cdots+a_{n} w^{n} \rightarrow a_{0}+a_{1} F_{1}(z)+\cdots+a_{n} F_{n}(z) .
$$

By a result of Kövári [8] and Anderson and Clunie [2], $T_{\rho}$ is a bounded operator and it can be uniquely extended to a bounded isomorphism between $A\left(\bar{U}_{\rho}\right)$ and $A\left(K_{\rho}\right)$; hence $T_{\rho}^{-1}$ is a bounded isomorphism between $A\left(K_{\rho}\right)$ and $A\left(\bar{U}_{\rho}\right)$. From the proofs of these results it also follows that the norms of $T_{\rho}$ and $T_{\rho}^{-1}$ are uniformly bounded, i.e.,

$$
\left\|T_{\rho}\right\|,\left\|T_{\rho}^{-1}\right\| \leq C, \quad r \leq \rho \leq R,
$$

for some constant $C$. 
Finally, if we consider $A\left(\bar{U}_{R}\right)$ as a linear subspace of $A\left(\bar{U}_{\rho}\right)$ then, on $A\left(\bar{U}_{R}\right)$, the mappings $T_{R}$ and $T_{\rho}$ coincide. This follows from the fact that they coincide on the set of polynomials, and the polynomials are dense in $A\left(\bar{U}_{R}\right)$.

Now let

$$
P_{n}^{*}(f, \cdot):=T_{R} \circ \tau_{n} \circ T_{R}^{-1}\left(Q_{n}(f, \cdot)\right),
$$

where $\tau_{n}$ was defined above and $Q_{n}(f, \cdot)$ was defined in (2.1).

From the boundedness of $T_{R}, T_{R}^{-1}$, and $\tau_{n}$ we get (cf. (7.2))

$$
\left\|P_{n}^{*}(f)\right\|_{K} \leq\left\|T_{R}\right\|\left\|\tau_{n}\right\|\left\|T_{R}^{-1}\right\|\left\|Q_{n}(f)\right\|_{K} \leq 3 C^{2} .
$$

For $\rho<R$ we have

$$
\begin{aligned}
\left\|P_{n}^{*}(f)-Q_{n}(f)\right\|_{K_{\rho}}=\left\|P_{n}^{*}(f)-Q_{n}(f)\right\|_{\gamma_{\rho}} \\
\quad=\left\|T_{R} \circ\left(\tau_{n}-I\right) \circ T_{R}^{-1}\left(Q_{n}(f)\right)\right\|_{\gamma_{\rho}}=\left\|T_{\rho} \circ\left(\tau_{n}-I\right) \circ T_{R}^{-1}\left(Q_{n}(f)\right)\right\|_{\gamma_{\rho}} \\
\quad \leq\left\|T_{\rho}\right\|_{A\left(\bar{U}_{\rho}\right) \rightarrow A\left(K_{\rho}\right)}\left\|\tau_{n}-I\right\|_{A\left(\bar{U}_{R}\right) \rightarrow A\left(\bar{U}_{\rho}\right)}\left\|T_{R}^{-1}\right\|_{A(K) \rightarrow A\left(\bar{U}_{R}\right)}\left\|Q_{n}(f)\right\|_{K} \\
\quad \leq C 4(\rho / R)^{n / 2} C \cdot 1=4 C^{2}(\rho / R)^{n / 2},
\end{aligned}
$$

where we used (7.1) and (7.2).

Our estimates show that for the polynomials

$$
p_{n}(z):=P_{n}(f, z)+E_{n}(f) P_{n}^{*}(f, z)
$$

we have

$$
\left\|f-p_{n}\right\|_{K} \leq\left(3 C^{2}+1\right) E_{n}(f)
$$

and

$$
\left\|f-p_{n}\right\|_{K_{\rho}} \leq 4 C^{2}\left(\frac{\rho}{R}\right)^{n / 2} E_{n}(f), \quad r<\rho<R,
$$

which proves Theorem 4 .

\section{Proof of Theorem 5}

Let $x_{1}, x_{2}, \ldots$ be countably many nonisolated points of $\partial K$ such that the set $\left\{x_{1}, x_{2}, \ldots\right\}$ is dense at every nonisolated point of $\partial K$ and $g_{1}, g_{2}, \ldots$ be an enumeration of all not identically zero polynomials with rational coefficients that have norm strictly less than 1 on $K$. Set

$$
S_{m, k, l, t}:=\left\{f \in A(K): \max _{z \in K,\left|z-x_{l}\right| \geq 1 / l}\left|Q_{n}(f, z)-g_{m}(z)\right| \geq \frac{1}{k} \text { for every } n\right\} \text {. }
$$

We have to show that the set

$$
S_{1}:=\bigcup_{m, k, l, l=1}^{\infty} S_{m, k, l, t}
$$


is of the first category in $A(K)$. Since for $l^{\prime}>l$ and $k^{\prime}>k$ we have $S_{m, k, l, t} \subseteq$ $S_{m, k^{\prime}, l^{\prime}, t}$, it follows that

$$
S_{1}=\bigcup\left\{S_{m, k, l, t}: \frac{1}{k}<\left\|g_{m}\right\|_{K \backslash U_{1 / l}\left(x_{t}\right)}, 1 \leq m, k, l, t<\infty\right\} .
$$

Exactly as was done in the proof of Theorem 1, one can easily see that each $S_{m, k, l, t}$ with $1 / k<\left\|g_{m}\right\|_{K \backslash U_{1 / l}\left(x_{t}\right)}$ is a closed subset of $A(K)$; hence if $S_{1}$ is not of the first category then some $S_{m, k, l, t}$ contains an $f_{0}$ together with a neighborhood of it. Approximating $f_{0}$ by a suitable polynomial, we get in the same manner as in the proof of Theorem 1 that if $S_{1}$ is not of the first category, then there exist $n_{0}, x_{t}, l, k$, and $m$ such that for every $F$ in $A(K)$

$$
\max _{\substack{\left|z-x_{i}\right| \geq 1 / l \\ z \in K}}\left|Q_{n_{0}}(F, z)-g_{m}(z)\right| \geq \frac{1}{k},
$$

and this is what we will disprove below. The contradiction thus obtained will prove Theorem 5 .

Let $z_{1}, \ldots, z_{n_{0}+2}$ be $n_{0}+2$ points from $U_{1 / l}\left(x_{t}\right)$ belonging to the set $\widetilde{K}$ defined in Lemma 1 . We have already seen in the proof of Theorem 1 that there are numbers $\xi_{1}, \ldots, \xi_{n_{0}+2}$ of absolute value 1 such that if $F \in A(K)$ with $\|F\|_{K}=1$ takes the values $\xi_{j}$ at $z_{j}$, then $P_{n_{0}}(F, z) \equiv 0$. We will also show that such an $F$ exists with the additional property that it approximates $g_{m}$ on $K \backslash U_{1 / l}\left(x_{t}\right)$ with error less than $1 / k$, and this will conclude the proof because for such $F$ we have $Q_{n_{0}}(F, z) \equiv F(z)$.

Since $g_{m}$ is a polynomial of norm less than 1, there is a neighborhood in C of $x_{t}$ where $g_{m}$ is less than 1 in absolute value. Without loss of generality we may assume $l$ so large that on $\overline{U_{1 / l}\left(x_{t}\right)}$ we have $\left|g_{m}\right|<1$. Consider the set $G_{0}$ constructed in the proof of Lemma 1 for the points $z_{1}, \ldots, z_{n_{0}+2}$. On $K \cup \overline{U_{1 / l}\left(x_{t}\right)}$ the function $g_{m}$ is continuous and smaller than 1 in absolute value; hence it has a continuous extension $g_{m}^{*}$ to $\bar{G}_{0}$ such that $\left\|g_{m}^{*}\right\|_{\bar{G}_{0}}<1$. In our construction below it will be of vital importance that this $g_{m}^{*}$ is analytic on $G_{0} \cap U_{1 / l}\left(x_{t}\right)$.

Since $\bar{G}_{0}$ contains $K$, it will be enough to show that a continuous $F$ exists on $\bar{G}_{0}$ that approximates $g_{m}^{*}$ on $G_{0} \backslash U_{1 / l}\left(x_{t}\right)$ with error less than $1 / k ; F\left(z_{j}\right)=$ $\xi_{j}, 1 \leq j \leq n_{0}+2 ;\|F\|_{\bar{G}_{0}} \leq 1$ and $F$ is analytic in $K^{0}$.

$G_{0}$ is mapped onto $U$ by a conformal mapping $\phi$ such that $\phi$ is a homeomorphism of $\bar{G}_{0}$ onto $\bar{U}$ (see the proof of Lemma 1). The image of $K^{0}$ will be the interior of $\phi(K)$, the image of $K$, and the image of $\bar{G}_{0} \cap U_{1 / l}\left(x_{t}\right)$ will contain neighborhoods relative to $\bar{U}$ of the points $w_{1}:=\phi\left(z_{1}\right), \ldots, w_{n_{0}+2}:=$ $\phi\left(z_{n_{0}+2}\right)$; furthermore $g_{m}^{*}$ is transformed into a continuous function $g$ defined on $\bar{U}$ which is holomorphic in $\phi\left(K^{0}\right)$ and in $\phi\left(G_{0} \cap U_{1 / l}\left(x_{t}\right)\right)$. Thus $g$ is holomorphic in a neigiborhood (relative to $U$ ) of each $w_{j}, 1 \leq j \leq n_{0}+2$. 
Hence, all we have to prove is the following: for every $\eta>0$ there exists a continuous function $f$ on $\bar{U}$ such that $f$ is holomorphic on $\phi\left(K^{0}\right), f\left(w_{j}\right)=$ $\xi_{j}, 1 \leq j \leq n_{0}+2$, and $|f(z)-g(z)|<\eta$ if $z \notin U_{\eta}\left(w_{j}\right), 1 \leq j \leq n_{0}+2$. In fact, then choosing $\eta$ so small that $\eta<1 / k$ and the inverse images of the sets $U_{\eta}\left(w_{j}\right) \cap U$ under $\phi$ are all contained in $U_{1 / l}\left(x_{t}\right)$ and setting $F(z)=f(\phi(z))$, we get the desired function $F$ on $K$.

We construct $f$ in several steps. For the sake of simpler notation let $n=$ $n_{0}+2$, and we can assume $\eta$ so small that in each set $U \cap U_{\eta}\left(w_{j}\right)$ the function $g$ is holomorphic and furthermore that the sets $U_{\eta}\left(w_{j}\right), 1 \leq j \leq n$, are pairwise disjoint.

Step 1. If $\eta>0$ and $w_{1}, \ldots, w_{n} \in \partial U$ are given, then there is a function $R$ in the disk algebra $\mathscr{A}=A(\bar{U})$ such that

(a) $|R(z)|<1, z \in \bar{U}$,

(b) $|R(z)-1|<\eta, z \in \bar{U} \backslash\left(\bigcup_{j=1}^{n} U_{\eta}\left(w_{j}\right)\right)$,

(c) $R\left(w_{j}\right)=R^{\prime}\left(w_{j}\right)=0,1 \leq j \leq n$,

hold.

Proof. For $c>0$ let

$$
R_{c}(z):=\exp (-c \sqrt{1 /(1-z)}),
$$

where we take that branch of $\sqrt{z}, z \notin(-\infty, 0)$, for which $\sqrt{1}=1$, and of course we set $R_{c}(1)=0$. Then $R_{c} \in \mathscr{A},\left|R_{c}(z)\right|<1$ for $z \in U$, and every derivative (taken on $U$ ) of $R_{c}$ vanishes at $z=1$. Thus, for small $c$,

$$
R(z):=\prod_{j=1}^{n} R_{c}\left(w_{j}^{-1} z\right)
$$

will satisfy properties $(a)-(c)$.

Step 2. With the above notation, for every $\eta>0$ there is a function $R^{*}$ continuous on $\bar{U}$ and holomorphic in $\phi\left(K^{0}\right) \cup\left(\bigcup_{j=1}^{n} U \cap U_{\eta}\left(w_{j}\right)\right)$ such that

(a) $\left|R^{*}(z)\right|<1, z \in \bar{U}$,

(b) $\left|R^{*}(z)-g(z)\right|<\eta, z \in \bar{U} \backslash\left(\bigcup_{j=1}^{n} U_{\eta}\left(w_{j}\right)\right)$,

(c) $R^{*}\left(w_{j}\right)=\left(R^{*}\right)^{\prime}\left(w_{j}\right)=0,1 \leq j \leq n$,

hold.

Proof. Just multiply the $R$ from Step 1 by $g$.

Step 3. For every $\eta>0$ there is an $f$ holomorphic in $\phi\left(K^{0}\right)$ with the properties

(a) $|f(z)|<1, z \in \bar{U} \backslash\left\{w_{1}, \ldots, w_{n}\right\}$,

(b) $|f(z)-g(z)|<\eta, \quad z \in \bar{U} \backslash\left(\bigcup_{j=1}^{n} U_{\eta}\left(w_{j}\right)\right)$,

(c) $f\left(w_{j}\right)=\xi_{j}, 1 \leq j \leq n$. 
By proving this we will complete the proof of Theorem 5 .

Proof. Let

$$
H_{j, m}(z):=\left(\frac{1+w_{j}^{-1} z}{2}\right)^{m} \prod_{\substack{k=1 \\ k \neq j}}^{n} \frac{\left(z-w_{k}\right)^{2}\left(z-w_{j}^{2} / w_{k}\right)^{2}}{\left(w_{j}-w_{k}\right)^{2}\left(w_{j}-w_{j}^{2} / w_{k}\right)^{2}} .
$$

Since each factor

$$
\left|\left(z-w_{k}\right)\left(z-\frac{w_{j}^{2}}{w_{k}}\right)\right|
$$

has a local maximum on $\partial U$ at $z=w_{j}$ and

$$
\left|\left(1+w_{j}^{-1} z\right) / 2\right|<1 \text { for } z \in \bar{U}, z \neq w_{j},
$$

for large $m$ we have

(i) $\left|H_{j, m}(z)\right|<1, z \in \bar{U} \backslash\left\{w_{j}\right\}$,

(ii) $H_{j, m}\left(w_{j}\right)=1$,

(iii) $\left|H_{j, m}(z)\right| \leq C\left|z-w_{k}\right|^{2}$ for $z \in \partial U, k \neq j, 1 \leq k \leq n$, where $C$ is independent of $m$, and

(iv) $\left|H_{j, m}(z)\right|<\eta_{m}, \quad z \in \bar{U} \backslash\left(\bigcup_{s=1}^{n} U_{\eta}\left(w_{s}\right)\right)$, where $\eta_{m} \rightarrow 0$ as $m \rightarrow \infty$.

Now let

$$
f(z):=f_{m}(z):=R^{*}(z)+\sum_{j=1}^{n} \xi_{j} H_{j, m}(z),
$$

for some large $m$, where $R^{*}$ is the function constructed in Step 2. Clearly, $f$ is continuous on $\bar{U}$ and holomorphic on $\phi\left(K^{0}\right) \cup\left(\bigcup_{j=1}^{n} U_{\eta}\left(w_{j}\right)\right)$. Property (c) is obvious for $f$ as well as property (b) for every large $m$ if we use (iv) above and property (b) of Step 2. Also, if $m$ is sufficiently large, then from (iv) and (a) of Step 2 we get

$$
|f(z)|<1 \quad \text { if } z \in \bar{U} \backslash \bigcup_{j=1}^{n} U_{\eta}\left(w_{j}\right) .
$$

Thus, the only thing we have to verify is that $|f(z)|<1$ if

$$
z \in\left(\overline{U_{\eta}\left(w_{j}\right)} \cap \bar{U}\right) \backslash\left\{w_{j}\right\}, \quad 1 \leq j \leq n .
$$

This is where we use the analyticity of $f$ in $U_{\eta}\left(w_{j}\right) \cap U$, which allows us to consider the corresponding inequality only on the boundary of $U_{\eta}\left(w_{j}\right) \cap U$. On $\partial U_{\eta}\left(w_{j}\right) \cap U$ we have $|f(z)|<1$ by (8.2). Thus, to conclude the proof we have to show a similar inequality for every large $m$ on $\left(\partial U \cap U_{\eta}\left(w_{j}\right)\right) \backslash\left\{w_{j}\right\}$.

By property (c) of Step 2 and property (iii) above there is a constant $C_{j}$ such that if $w_{j}=e^{i \psi_{j}}$, then for every $\varphi$ and $m$,

$$
\left|R^{*}\left(e^{i \varphi}\right)+\sum_{\substack{k=1 \\ k \neq j}}^{n} \xi_{k} H_{k, m}\left(e^{i \varphi}\right)\right| \leq C_{j}\left|\varphi-\psi_{j}\right|^{2}
$$


We can assume $C_{j}$ is so large that for $\left|\varphi-\psi_{j}\right|<1 /\left(2\left(C_{j}+1\right)\right)$, the expression in (8.1) with $z=e^{i \varphi}$ is not bigger than its value at $\varphi=\psi_{j}$. But then, for $0<\left|\varphi-\psi_{j}\right|<1 /\left(2\left(C_{j}+1\right)\right)$, we have

$$
\left|H_{j, m}\left(e^{i \varphi}\right)\right| \leq\left|\frac{1+e^{i\left(\varphi-\psi_{j}\right)}}{2}\right|^{m}=\left(\cos \frac{\varphi-\psi_{j}}{2}\right)^{m},
$$

which is smaller than $1-C_{j}\left|\varphi-\psi_{j}\right|^{2}$ if $m$ is large enough. Thus, for such $z=e^{i \varphi}$ we get $|f(z)|<1$ from (8.3). On the other hand, (a) from Step 2 and (i) from above imply that if $z=e^{i \varphi} \in U_{\eta}\left(w_{j}\right)$ and $\left|\varphi-\psi_{j}\right| \geq 1 /\left(2\left(C_{j}+1\right)\right)$, then for large $m$ we have $|f(z)|=\left|f_{m}(z)\right|<1$ and the proof is complete.

\section{Proof of Theorem 6}

We start with a lemma.

Lemma 2. Let $K$ be a compact set with connected complement. There exists a subset $\widetilde{\widetilde{K}}$ of $\partial K$ with the following properties: $\widetilde{\widetilde{K}}$ is dense in $\partial K$ and if $z_{1}, \ldots, z_{n} \in \widetilde{\widetilde{K}}$ are arbitrary points and $\xi_{1}, \ldots, \xi_{n}$ are arbitrary numbers of modulus 1 , then there is a polynomial $P$ such that

(a) $|P(z)|<1, \quad z \in K \backslash\left\{z_{1}, \ldots, z_{n}\right\}$,

(b) $P\left(z_{j}\right)=\xi_{j}, 1 \leq j \leq n$, and

(c) $1-|P(z)|$ has finite order zeros at $z_{1}, \ldots, z_{n}$.

The last assertion means that there is a $\delta>0$ and $\alpha$ such that for $\left|z-z_{j}\right|<$ $\delta, z \in K$, we have $1-|P(z)| \geq\left|z-z_{j}\right|^{\alpha}$. We warn the reader that property (c) is not automatic; (a) and (b) do not imply (c) even for linear polynomials.

Proof. First we define $\widetilde{\widetilde{K}}$. Let $\widetilde{\widetilde{K}}$ be the set of all $w \in \partial K$ such that there is a polynomial $P$ that takes the value 1 at $w$ and at every other point of $K$ the absolute value of $P$ is smaller than 1 .

Next we prove that $\widetilde{\widetilde{K}}$ is dense in $\partial K$. Let $z_{0} \in \partial K$ and $\varepsilon>0$ be arbitrary, and pick a $u$ in the complement of $K$ and a $\rho<\varepsilon / 4$ such that $\left|z_{0}-u\right|<\varepsilon / 4$ and $\overline{U_{\rho}(u)} \cap K=\varnothing$. The function $g(z):=1 /(z-u)$ is holomorphic and one-toone on $\mathbf{C} \backslash\{u\}$ and, since $K$ has connected complement, $g$ can be uniformly approximated by polynomials on a compact set containing $K$ in its interior (e.g., see the proof of Lemma 1). If the approximation is fine enough, then the approximating polynomial $P_{0}$ will also be one-to-one on $K$ (see the proof of Theorem 2); furthermore $\left|P_{0}\right|$ will attain its maximum on $K$ at some point $w$ in $U_{\varepsilon}\left(z_{0}\right)$ because $|g(z)|=1 /|z-u|$ does so on $U_{\varepsilon / 2}\left(z_{0}\right)$. It is easy to check that then the polynomial

$$
P_{u}(z):=\frac{1}{2}\left(P_{0}(w)^{-1} P_{0}(z)+1\right)
$$

has the properties $P_{w}(w)=1$ and $\left|P_{w}(z)\right|<1$ for $z \in K \backslash\{w\}$. Thus $w \in$ $\widetilde{\widetilde{K}} \cap U_{\varepsilon}\left(z_{0}\right)$ and the density of $\widetilde{\widetilde{K}}$ in $\partial K$ is proved. 
Now let $z_{1}, \ldots, z_{n} \in \widetilde{\widetilde{K}}$ and $\left|\xi_{1}\right|=\cdots=\left|\xi_{n}\right|=1$ be arbitrary. Then for each $j$ there is a polynomial $p_{j}$ with the property $p_{j}\left(z_{j}\right)=1$ and $\left|p_{j}(z)\right|<1$ for $z \in K \backslash\left\{z_{j}\right\}$. We set $w_{j i}:=p_{j}\left(z_{i}\right)$. It is easy to construct a polynomial $Q$ such that $Q(1)=1, Q\left(w_{j i}\right)=0$ for $1 \leq i, j \leq n, i \neq j$, and $|Q(w)|<1$ for every $|w| \leq 1, w \neq 1$. In fact, for some large $k$,

$$
Q(w)=\left(\frac{1+w}{2}\right)^{k} \prod_{i \neq j} \frac{\left(w-w_{j i}\right)\left(w-\bar{w}_{j i}\right)}{\left(1-w_{j i}\right)\left(1-\bar{w}_{j i}\right)}
$$

will satisfy these requirements (see the proof of Theorem 5).

Now define

$$
P(z) \equiv P_{m}(z):=\sum_{j=1}^{n} \xi_{j}\left[Q\left(p_{j}(z)\right) \frac{1+p_{j}(z)}{2}\right]^{m}
$$

for some large $m$. We claim that $P$ satisfies (a)-(c) above if $m$ is sufficiently large.

Property (b) follows from the definitions of the $p_{j}$ 's and $Q$. Next we prove (c). Since the $p_{j}$ 's are polynomials, we can choose a $\beta$ and a $\delta>0$ such that if $\left|z-z_{j}\right|<\delta$ for some $j$, then $\left|1-p_{j}(z)\right| \geq\left|z-z_{j}\right|^{\beta}$. But then

$$
\begin{aligned}
1-\left|Q\left(p_{j}(z)\right) \frac{1+p_{j}(z)}{2}\right|^{m} & \geq 1-\left|\frac{1+p_{j}(z)}{2}\right|^{2} \\
& \geq \frac{\left|1-p_{j}(z)\right|^{2}}{4} \geq \frac{1}{4}\left|z-z_{j}\right|^{2 \beta}
\end{aligned}
$$

and this easily proves (c) for every $m>4 \beta+2$. In fact, there is a constant $C$ such that independently of $1 \leq i, j \leq n, i \neq j$, and $z \in K$

$$
\left|Q\left(p_{i}(z)\right)\left(1+p_{i}(z)\right) / 2\right| \leq C\left|z-z_{j}\right| \text {. }
$$

But then for

$$
\left|z-z_{j}\right|<\min \left(C^{-2},(8 n)^{-1}\right)
$$

and $m>4 \beta+2$ we get

$$
\begin{aligned}
\left|Q\left(p_{i}(z)\right)\left(1+p_{i}(z)\right) / 2\right|^{m} & \leq\left(C\left|z-z_{j}\right|\right)^{m} \leq\left|z-z_{j}\right|^{m / 2} \\
& <(1 / 8 n)\left|z-z_{j}\right|^{2 \beta}, \quad 1 \leq i \leq n, i \neq j,
\end{aligned}
$$

and this, together with (9.1), proves (c).

Finally, for $z \in K \backslash\left\{z_{1}, \ldots, z_{n}\right\}$ satisfying (9.2) property (a) follows from (9.1) and (9.3). On the other hand, for other points $z \in K \backslash\left\{z_{1}, \ldots, z_{n}\right\}$,

$$
\left|Q\left(p_{j}(z)\right)\left(1+p_{j}(z)\right) / 2\right| \leq \tau, \quad 1 \leq j \leq n,
$$

for some $\tau<1$ independent of $z$, and so for $m>(\log n) /(\log 1 / \tau)$ we also get $|P(z)|<1$.

In the proof of Theorem 6 we need the following modification of Lemma 2. 
Lemma 3. For the subset $\widetilde{\widetilde{K}}$ of $\partial K$ defined in Lemma 2 above the following is true. Suppose $u$ is a nonnegative continuous function on $K$ that vanishes only at the points $w_{1}, \ldots, w_{k}$ belonging to $\widetilde{\widetilde{K}}$ and satisfies

$$
u(z) \geq\left|z-w_{j}\right|^{\beta}, \quad\left|z-w_{j}\right| \leq \delta, 1 \leq j \leq k,
$$

for some $\delta>0$ and $\beta$. Furthermore let $\left\{z_{1}, \ldots, z_{n}\right\} \subseteq \widetilde{\widetilde{K}}$ be any finite set disjoint from $\left\{w_{1}, \ldots, w_{k}\right\}$ and $\xi_{1}, \ldots, \xi_{n}$ be any numbers of modulus 1 . Then for $\varepsilon>0$ there is a polynomial $P$ with the following properties:

(a) $|P(z)| \leq \varepsilon u(z), \quad z \in K$,

(b) $P\left(z_{j}\right)=\|P\|_{K} \xi_{j}, 1 \leq j \leq n$,

(c) $|P(z)|<\|P\|_{K}$ if $z \in K \backslash\left\{z_{1}, \ldots, z_{n}\right\}$, and

(d) $\|P\|_{K}-|P(z)|$ has finite order zeros at $z_{1}, \ldots, z_{n}$.

We remark that property $(\mathrm{d})$ will be crucial in our construction below because we will repeatedly apply Lemma 3 , and the $u$ will be the minimum of $\|P\|-$ $|P(z)|$ for the $P$ 's constructed up to the step in question. Thus, (d) will ensure that the assumptions on $u$ will be passed on to the next step.

We also mention that all the essential features of the proof are contained in the proof of Lemma 2 above and actually the present lemma is the variant of Lemma 2 needed to prove Theorem 6. However, we regard it instructive to separate the content of the proof as we did above because Lemma 3 is of a rather technical flavor.

Proof. Consider the construction given in Lemma 2 with the modification that now the polynomial $Q$ also vanishes at every $p_{j}\left(w_{t}\right), 1 \leq t \leq k, 1 \leq j \leq$ $n$. If $P_{0}(t):=P_{0, m}(t)$ is the polynomial we get from Lemma 2 with obvious modifications in the proof (recall that the construction involved a parameter $m$, and $m$ could be any sufficiently large number), then set $P(z):=\rho P_{0}(z)$ for some small $\rho>0$. By the proof of Lemma 2 (see especially the analogs of (9.1), (9.3), and (9.4)), properties (a) through (d) will hold for every sufficiently small $\rho$ and large $m$.

After these preliminaries we finally turn to the construction of a function $f$ for which the measures $\nu_{n}(f)$ have every possible limit distribution.

We construct three sequences, polynomials $\left\{g_{k}\right\}$, integers $\left\{n_{k}\right\}$, and sets $\left\{H_{k}\right\}$, as follows. Let $g_{1}, n_{1}, H_{1}$ be arbitrarily chosen to satisfy the requirements below. Suppose the polynomial $g_{k-1}$ and the set $H_{k-1}$ have already been chosen for some $k$ and assume that $\left|g_{k-1}\right|$ attains its maximum on $K$ exactly at the points of $H_{k-1}$ and $\left\|g_{k-1}\right\|_{K}-\left|g_{k-1}(z)\right|$ has finite order zeros at the points of $H_{k-1}$ (and of course we assume these properties for every $k^{\prime}<k$ as well). Furthermore, we assume that $n_{k-1}=\left|H_{k-1}\right|-2$. We are going to construct $g_{k}, n_{k}$, and $H_{k}$ with the same properties; furthermore, $g_{k}$ will vanish on every $H_{k^{\prime}}, k^{\prime}<k$. 
In Lemma 3 we set

$$
u(z) \equiv u_{k}(z):=\lim _{1 \leq s \leq k-1}\left\{\left\|g_{s}\right\|_{K}-\left|g_{s}(z)\right|\right\} .
$$

Let $n_{k}$ be an integer larger than the degrees of $g_{1}, \ldots, g_{k-1}$, and pick $H_{k}=$ $\left\{z_{1}^{(k)}, \ldots, z_{n_{k}+2}^{(k)}\right\} \subseteq \widetilde{\widetilde{K}} \backslash\left(\bigcup_{s=1}^{k-1} H_{s}\right)$ for the moment arbitrarily. There are numbers $\xi\left(z_{1}^{(k)}\right), \ldots, \xi\left(z_{n_{k}+2}^{(k)}\right)$ of modulus 1 such that if $g$ is a function in $A(K)$ with $g\left(z_{i}^{(k)}\right)=\xi\left(z_{i}^{(k)}\right)\|g\|_{K}, 1 \leq i \leq n_{k}+2$, then zero is its best approximant out of $\Pi_{n_{k}}$ (see the proof of Theorem 1). Now let $g_{k}$ be the polynomial function constructed in Lemma 3 for $u(z)=u_{k}(z), \varepsilon=\varepsilon_{k}=2^{-k}, z_{i}=z_{i}^{(k)}$, $n=n_{k},\left\{w_{1}, \ldots\right\}=\bigcup_{s=1}^{k-1} H_{s}$, and $\xi_{i}=\xi\left(z_{i}^{(k)}\right)$. With this choice the construction of the sets $H_{1}, H_{2}, \ldots$ and the polynomials $g_{1}, g_{2}, \ldots$ is complete, and notice that the construction can be carried out because Lemma 3 ensures that the properties we assumed about $g_{k-1}$ and $H_{k-1}$ will continue to hold for $g_{k}$ and $H_{k}$.

Set

$$
f(z):=\sum_{k=1}^{\infty} g_{k}(z)
$$

Since $\left\|g_{k}\right\|_{K} \leq$ const. $\varepsilon_{k}=$ const. $2^{-k}$, the function $f$ belongs to $A(K)$. We claim that for every $k \geq 2$ the best approximant of $f$ out of $\Pi_{n_{k}}$ is $\sum_{j=1}^{k-1} g_{j}$ and that $H_{n_{k}}(f)=H_{k}$. In fact,

$$
f(z)-\sum_{j=1}^{k-1} g_{j}(z)=g_{k}(z)+\sum_{j=k+1}^{\infty} g_{j}(z),
$$

and by the construction we get for every $z \in K \backslash H_{k}$

$$
\sum_{j=k+1}^{\infty}\left|g_{j}(z)\right| \leq\left(\left\|g_{k}\right\|_{K}-\left|g_{k}(z)\right|\right) \sum_{j=k+1}^{\infty} 2^{-j}<\left\|g_{k}\right\|_{K}-\left|g_{k}(z)\right|,
$$

and so

$$
\left|f(z)-\sum_{j=1}^{k-1} g_{j}(z)\right|<\left\|g_{k}\right\| \quad \text { for } z \in K \backslash H_{k} .
$$

On the other hand, every $g_{j}, j>k$, vanishes on $H_{k}$; therefore

$$
f\left(z_{s}^{(k)}\right)-\sum_{j=1}^{k-1} g_{j}\left(z_{s}^{(k)}\right)=g_{k}\left(z_{s}^{(k)}\right)=\xi\left(z_{s}^{(k)}\right)\left\|g_{k}\right\|_{K},
$$

and the choice of $\xi\left(z_{s}^{(k)}\right), 1 \leq s \leq n_{k}+2$, implies that zero is the best approximation of $f-\sum_{j=1}^{k-1} g_{j}$ out of $\Pi_{n_{k}}$. From (9.5) and (9.6) we see that indeed $H_{n_{k}}(f)=H_{k}$. 
Finally, we can complete the proof of Theorem 6 as follows. In the construction above the sets $H_{k}$ were arbitrary $\left(n_{k}+2\right)$-point subsets of the sets $\widetilde{\widetilde{K}} \backslash\left(\bigcup_{j=1}^{k-1} H_{j}\right), \quad k=2,3, \ldots$, and the latter sets are dense at every nonisolated point of $\partial K$. This means that we get as weak-star limits of the measures $\nu_{n_{k}}(f)$ all those measures that are limit distributions of a subsequence of $H_{1}, \ldots, H_{k}, \ldots$ and at the same time the choice of $H_{k}$ is completely at our disposal from a (in $\partial K \backslash K_{\text {isolated }}$ ) dense set. Clearly, we can choose $H_{1}, \ldots$ so that every finite discrete probability distribution with rational values and with support in a countable dense subset of $\partial K \backslash K_{\text {isolated }}$ (there are countably many such measures if the latter set is fixed) is the limit distribution of some subsequence of the $H_{k}$ 's, and since these measures are weak-star dense in the space of probability measures on $\partial K \backslash K_{\text {isolated }}$, we get every possible limit distribution if we choose $H_{1}, H_{2}, \ldots$ suitably.

\section{REFERENCES}

1. S. J. Al'per, Asymptotic values of best approximation of analytic functions in a complex domain, Uspekhi Mat. Nauk 14 (1959), 131-134.

2. J. M. Anderson and J. Clunie, Isomorphisms of the disc algebra and inverse Faber sets, Math. Z. 188 (1985), 545-558.

3. H.-P. Blatt, E. B. Saff, and M. Simkani, Jentzsch-Szegö type theorems for the zeros of best approximants, J. London Math. Soc. 38 (1988), 307-316.

4. H.-P. Blatt, E. B. Saff, and V. Totik, The distribution of extreme points in best complex polynomial approximation, Constr. Approx. 5 (1989), 357-370.

5. G. M. Goluzin, Geometric theory of functions of a complex variable, Transl. Math. Monographs, vol. 26, Amer. Math. Soc., Providence, R.I., 1969.

6. K. Hoffman, Banach spaces of analytic functions, Prentice-Hall, London, 1962.

7. M. I. Kadec, On the distribution of points of maximal deviation in the approximation of continuous functions by polynomials, Uspekhi Mat. Nauk 15 (1960), 199-202.

8. T. Kövári, On the order of polynomial approximation for closed Jordan domains, J. Approx. Theory 5 (1972), 362-373.

9. A. Kroó and E. B. Saff, The density of extreme points in complex polynomial approximation, Proc. Amer. Math. Soc. 103 (1988), 203-209.

10. A. I. Markushevich, Theory of functions of a complex variable, vol. III, Prentice-Hall, Englewood Cliffs, N.J., 1967.

11. A. Pinkus, n-widths in approximation theory, Springer-Verlag, Berlin, 1985.

12. W. Rudin, Real and complex analysis, 2nd ed., McGraw-Hill, New York, 1974.

13. E. B. Saff, A principle of contamination in best polynomial approximation, Approximation and Optimization, Lecture Notes in Math., vol. 1354 (Gomez, Guerra, Jimeniz, Lopez, eds.), Springer-Verlag, Heidelberg, 1988, pp. 79-97.

14. E. B. Saff and V. Totik, Limitations of the $C F$ method for polynomial approximation, J. Approx. Theory (to appear).

15. __ Polynomial approximation to piecewise analytic functions, J. London Math. Soc. (to appear).

16. H. S. Shapiro, Topics in approximation theory, Lecture Notes in Math., vol. 187, SpringerVerlag, Berlin, 1971.

17. A. F. Timan, Theory of approximation of functions of a real variable, Hindustan, Delhi, 1966. 
18. L. N. Trefethen, Near-circularity of the error curve in complex Chebyshev approximation, J. Approx. Theory 31 (1981), 344-367.

19. L. N. Trefethen and M. H. Gutknecht, The Carathéodory-Fejér method for real rational approximation, SIAM J. Numer. Anal. 20 (1983), 420-436.

20. J. L. Walsh, Interpolation and approximation by rational functions in the complex domain, 5 th ed., Amer. Math. Soc. Colloq. Publ., vol. 20, Amer. Math. Soc., Providence, R.I., 1969.

Institute for Constructive Mathematics, Department of Mathematics, University OF SOUth Florida, TAMPa, Florida 33620-5700

Bolyai Institute, 6720 Szeged, Hungary 